\title{
Cost-effectiveness of Biological Disease-modifying Antirheumatic Drugs for the Treatment of Rheumatoid Arthritis: Implications for Clinical Practice
}

In this issue of The Journal, Stevenson, et al present an economic model on the cost-effectiveness of biological disease-modifying antirheumatic drugs (bDMARD) for rheumatoid arthritis (RA) in patients who can tolerate methotrexate $(\mathrm{MTX})^{1}$. Their work formed part of the evidence used by the UK National Institute for Health and Care Excellence (NICE) to update the technology appraisal guidance on the application of bDMARD in RA. In England, because prescription of bDMARD is restricted to patients with RA who have failed at least 2 conventional DMARD and have high disease activity [28-joint Disease Activity Score $($ DAS28) $>5.1]$, there was specific interest in the question of whether prescriptions should also be extended to those with moderate disease. The model presented is based on the healthcare setting in England and compares the continuous administration of nonbiological therapy to treatment with sequences of bDMARD in patients with moderate and moderate-to-severe RA. The authors report that the cost per quality-adjusted life-year (QALY) gained by bDMARD strategies over nonbiological DMARD treatment only was $£ 41,600$ (US $\$ 51,628$ ) for patients with severe RA and $£ 51,100$ for patients with moderate to severe RA, a cost well beyond the generally accepted NICE threshold of $£ 20,000-£ 30,000$. In subanalyses, restricting biologicals to patients with the worst prognosis with nonbiological therapy only, more favorable cost-effectiveness ratios were found: $£ 25,300$ per QALY for severe and $£ 28,500$ per QALY for moderate to severe RA. Hence the authors conclude that the cost-effectiveness of the administration of bDMARD in RA in England is questionable and meets currently accepted levels only in subsets of patients with the worst prognoses. In the updated NICE guidelines ${ }^{2}$, the eligibility criteria for bDMARD prescription remained unchanged and were not extended to include those with moderate RA.

What are the implications of this conclusion on clinical practice? Based on the threshold for cost-effectiveness alone, one would conclude that bDMARD treatment should not be administered on a regular basis to every RA patient with active disease. And if administered, bDMARD should be restricted to those patients with the worst prognosis of RA. However, before accepting such a conclusion and consequently introducing such a restriction, we should be convinced of the reliability of the results.

In the era before the emergence of biologicals, RA was characterized by its progressive nature, eventually resulting in irreversible joint damage with associated disability ${ }^{3}$. Progressive erosive disease with disabling joint malformations has now become a rare event due to a change in treatment strategies, i.e., early and intensive treatment combined with a treat-to-target strategy ${ }^{4}$. However, since up to $40 \%$ of patients still fail to show an adequate response taking conventional DMARD alone ${ }^{5}$, the additive success should be attributed to the availability of bDMARD as well ${ }^{3}$. Moreover, when bDMARD were given as induction therapy, remission rates up to $80 \%$ were reported ${ }^{6}$.

Because bDMARD treatment is expected to have a beneficial effect on treatment outcomes and quality of life, several aspects of an economic study questioning its cost-effectiveness need to be discussed: First, the validity of the model. If unrealistic assumptions were made in the modeling process, or if data from a nonrepresentative sample were used to populate the model, effect estimates could be expected to be biased and might not reflect the real-world situation. In this particular case, the authors deserve credit for a very thorough job of gathering the most representative data and effect estimates, which were used as input for the model. A network metaanalysis was conducted to estimate the relative effectiveness of the different bDMARD. Patient characteristics and expected time taking biologicals were obtained from British biological registries. The expected Health Assessment Questionnaire (HAQ) progression for patients taking nonbiological DMARD was estimated from a study using historical data from the 1980s and 1990s ${ }^{7}$. One could argue that these historical data do not reflect the HAQ progression we would observe today. However, owing to the paradigm changes of early, intensive, and tight-controlled treatment, patients today would be expected to show a more

See Cost-effectiveness of bDMARD, page 973

Personal non-commercial use only. The Journal of Rheumatology Copyright (C) 2017. All rights reserved. 
favorable course of disease than in the past, making the bDMARD even less cost-effective.

Apart from uncertainties in the modeling of the HAQ itself, another potential problem lies in correctly estimating health-related quality of life (EQ-5D) from HAQ levels. In the model, the EQ-5D was derived both directly from HAQ and indirectly, using pain as an intermediate. As the authors acknowledge in the full report, the relationship between HAQ and pain is uncertain and could have greatly influenced the incremental cost-effectiveness ratio (ICER). Another potential bias when deriving quality of life from HAQ could be that the HAQ may inherently measure 2 things: transient disability due to active inflammation and permanent disability due to irreversible joint damage. Whether a 1-point increase in either type of disability results in a similar reduction in quality of life is questionable. However, this is implicitly assumed in the model, where in the conventional DMARD group a progressive increase in HAQ is assumed, while in the bDMARD group HAQ progression is assumed to be constant.

Another assumption that is made for the bDMARD group, which may not be completely realistic, is that any reduction in HAQ is lost on failure of the bDMARD. This suggests that the potential for a disease-modifying effect by an early intensive (biological) intervention is not factored into the model. If such a benefit exists and were included in the model, this would favor the cost-effectiveness of biological treatment.

A last important issue that could influence the ICER is that only direct medical costs were included in the model. Indirect costs, such as costs due to productivity loss, were not taken into consideration and could potentially adjust the ICER in favor of biological treatment. Whether indirect costs should be taken into consideration in cost-effectiveness analyses is a matter of ongoing debate $8,9,10$. However, part of the problem with including productivity costs here would be that, to make a fair comparison, one would need to take into account the effect of productivity loss in the interventions displaced by purchasing the bDMARD therapy as well, which is unknown ${ }^{11}$.

As a final note, the setting needs to be considered. The analyses were performed taking the English healthcare setting as the standpoint, and models were populated using data obtained from British biologic registers. However, in contrast to most other countries, prescription of bDMARD in England is restricted to RA patients with high disease activity (DAS28 > 5.1), limiting the generalizability of results to different healthcare systems.

Despite these uncertainties, the relative costs of bDMARD will likely remain high. A cost-effectiveness study by Joensuu, et $a l^{12}$, in which a different modeling approach was used, reached similar conclusions. Further, even in an optimistic scenario in which bDMARD treatment would fall just below the cost-effectiveness threshold of $£ 20,000-£ 30,000$, the effect on healthcare budgets would remain considerable because of the high prevalence of the disease. However, several actions could be taken to substantially reduce these costs. First, the current biological treatment could be provided more efficiently by dosage reduction in patients whose disease is in remission. Results from several studies suggest that biological treatment can be reduced to a half dose in patients in remission without an increased risk of flare ${ }^{13,14}$. In some patients, biological treatment may even be withdrawn completely ${ }^{15,16}$, although identification of these patients, based on clinical factors, is not yet possible. A second substantial cost reduction may be realized by switching patients to less-expensive biosimilars. Studies comparing the effectiveness of biosimilars to their traditional counterparts find no difference in effectiveness or safety profiles ${ }^{17}$, suggesting that therapies can be safely exchanged. Combining the 2 strategies could yield even greater reductions in costs.

In this way, healthcare resources can be made available to other patients, while patients with RA can still benefit from biological treatment in a cost-effective way.

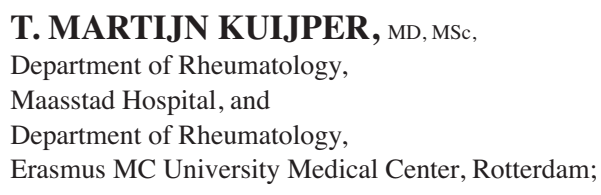
LEANDER R. BUISMAN, PhD, Institute of Health Policy and Management, Erasmus University, Rotterdam;

JOHANNA M. HAZES, MD, PhD, Department of Rheumatology, Erasmus MC University Medical Center, Rotterdam; ANGELIQUE E. WEEL, $M D, P h D$, Department of Rheumatology, Maasstad Hospital, and Erasmus MC University Medical Center, Rotterdam, the Netherlands.

Address correspondence to Dr. T.M. Kuijper, Maasstad Hospital, Department of Rheumatology, Postbus 9100, 3007 AC Rotterdam, The Netherlands; E-mail: kuijpert@maasstadziekenhuis.nl

\section{REFERENCES}

1. Stevenson MD, Wailoo AJ, Tosh JC, Hernandez-Alava M, Gibson LA, Stevens JW, et al. The cost-effectiveness of sequences of biological disease-modifying antirheumatic drug treatment in England for patients with rheumatoid arthritis who can tolerate methotrexate. J Rheumatol 2017;44:973-80.

2. UK National Institute for Health and Care Excellence. Adalimumab, etanercept, infliximab, certolizumab pegol, golimumab, tocilizumab and abatacept for rheumatoid arthritis not previously treated with DMARDs or after conventional DMARDs only have failed technology appraisal guidance [TA375]. 2016 [Internet. Accessed March 8, 2017.] Available from: www.nice.org.uk/ta375

3. Schett G, Emery P, Tanaka Y, Burmester G, Pisetsky DS, Naredo E, et al. Tapering biologic and conventional DMARD therapy in rheumatoid arthritis: current evidence and future directions. Ann Rheum Dis 2016;75:1428-37.

4. McInnes IB, O'Dell JR. State-of-the-art: rheumatoid arthritis. Ann

Personal non-commercial use only. The Journal of Rheumatology Copyright $\odot$ 2017. All rights reserved. 
Rheum Dis 2010;69:1898-906.

5. de Jong PH, Hazes JM, Han HK, Huisman M, van Zeben D, van der Lubbe PA, et al. Randomised comparison of initial triple DMARD therapy with methotrexate monotherapy in combination with low-dose glucocorticoid bridging therapy; 1-year data of the tREACH trial. Ann Rheum Dis 2014;73:1331-9.

6. Bijlsma JW, Welsing PM, Woodworth TG, Middelink LM, Petho-Schramm A, Bernasconi C, et al. Early rheumatoid arthritis treated with tocilizumab, methotrexate, or their combination (U-Act-Early): a multicentre, randomised, double-blind, double-dummy, strategy trial. Lancet 2016;388:343-55.

7. Norton S, Sacker A, Dixey J, Done J, Williams P, Young A, et al. Trajectories of functional limitation in early rheumatoid arthritis and their association with mortality. Rheumatology 2013;52:2016-24.

8. Krol M, Brouwer W, Rutten F. Productivity costs in economic evaluations: past, present, future. Pharmacoeconomics 2013; 31:537-49.

9. Jonsson B. Ten arguments for a societal perspective in the economic evaluation of medical innovations. Eur J Health Econ 2009; 10:357-9.

10. Claxton K, Palmer S, Sculpher M, Walker S. Appropriate perspectives for health care decisions. York: University of York; 2010.

11. Stevenson M, Archer R, Tosh J, Simpson E, Everson-Hock E, Stevens J. Adalimumab, etanercept, infliximab, certolizumab pegol, golimumab, tocilizumab and abatacept for the treatment of rheumatoid arthritis not previously treated with disease-modifying antirheumatic drugs and after the failure of conventional disease-modifying antirheumatic drugs only: systematic review and economic evaluation. Health Technol Assess 2016;20:1-610
12. Joensuu JT, Huoponen S, Aaltonen KJ, Konttinen YT, Nordstrom D, Blom M. The cost-effectiveness of biologics for the treatment of rheumatoid arthritis: a systematic review. PLoS One 2015;10:e0119683.

13. Emery P, Hammoudeh M, FitzGerald O, Combe B, Martin-Mola E, Buch $\mathrm{MH}$, et al. Sustained remission with etanercept tapering in early rheumatoid arthritis. N Engl J Med 2014;371:1781-92.

14. Smolen JS, Nash P, Durez P, Hall S, Ilivanova E, Irazoque-Palazuelos F, et al. Maintenance, reduction, or withdrawal of etanercept after treatment with etanercept and methotrexate in patients with moderate rheumatoid arthritis (PRESERVE): a randomised controlled trial. Lancet 2013;381:918-29.

15. Kuijper TM, Lamers-Karnebeek FB, Jacobs JW, Hazes JM, Luime JJ. Flare rate in patients with rheumatoid arthritis in low disease activity or remission when tapering or stopping synthetic or biologic DMARD: a systematic review. J Rheumatol 2015;42:2012-22.

16. Ghiti Moghadam M, Vonkeman HE, Ten Klooster PM, Tekstra J, van Schaardenburg D, Starmans-Kool M, et al. Stopping tumor necrosis factor inhibitor treatment in patients with established rheumatoid arthritis in remission or with stable low disease activity: a pragmatic multicenter, open-label randomized controlled trial. Arthritis Rheumatol 2016;68:1810-7.

17. Komaki Y, Yamada A, Komaki F, Kudaravalli P, Micic D, Ido A, et al. Efficacy, safety and pharmacokinetics of biosimilars of anti-tumor necrosis factor- $\alpha$ agents in rheumatic diseases; A systematic review and meta-analysis. J Autoimmun 2017 Feb 13 (E-pub ahead of print).

J Rheumatol 2017;44:965-7; doi:10.3899/jrheum.170334 